\title{
Composition of organics on asteroid (101955) Bennu
}

\author{
H. H. Kaplan ${ }^{1} \odot$, A. A. Simon ${ }^{1}$, V. E. Hamilton ${ }^{2}$, M. S. Thompson ${ }^{3}$, S. A. Sandford ${ }^{4}$, M. A. Barucci ${ }^{5}$, E. A. Cloutis ${ }^{6}$, \\ J. Brucato ${ }^{7}$, D. C. Reuter ${ }^{1}$, D. P. Glavin ${ }^{1}$, B. E. Clark ${ }^{8}$, J. P. Dworkin ${ }^{1}$, H. Campins ${ }^{9}$, J. P. Emery ${ }^{10}$, S. Fornasier ${ }^{5,11}$,
} X. D. Zou ${ }^{12}$, and D. S. Lauretta ${ }^{13}$

1 NASA Goddard Space Flight Center, Greenbelt, MD, USA e-mail: hannah.kaplan@nasa.gov

2 Southwest Research Institute, Boulder, CO, USA

3 Department of Earth, Atmospheric, and Planetary Sciences, Purdue University, West Lafayette, IN, USA

${ }^{4}$ NASA Ames Research Center, Moffett Field, CA, USA

5 LESIA, Observatoire de Paris, Université PSL, CNRS, Université de Paris, Sorbonne Université, 5 Place Jules Janssen, 92195 Meudon, France

6 Department of Geography, University of Winnipeg, Winnipeg, Canada

7 INAF-Arcetri Astrophysical Observatory, Florence, Italy

8 Department of Physics, Ithaca College, Ithaca, NY, USA

9 Department of Physics, University of Central Florida, Orlando, Florida, USA

10 Northern Arizona University, Flagstaff, AZ, USA

11 Institut Universitaire de France (IUF), Paris, France

12 Planetary Science Institute, Tucson, AZ, USA

13 Lunar and Planetary Laboratory, University of Arizona, Tucson, AZ, USA

Received 23 April 2021 / Accepted 30 July 2021

\section{ABSTRACT}

\begin{abstract}
Context. The Origins, Spectral Interpretation, Resource Identification, and Security-Regolith Explorer (OSIRIS-REx) mission detected an infrared absorption at $3.4 \mu \mathrm{m}$ on near-Earth asteroid (101955) Bennu. This absorption is indicative of carbon species, including organics, on the surface.

Aims. We aim to describe the composition of the organic matter on Bennu by investigating the spectral features in detail.

Methods. We use a curated set of spectra acquired by the OSIRIS-REx Visible and InfraRed Spectrometer that have features near $3.4 \mu \mathrm{m}(3.2$ to $3.6 \mu \mathrm{m})$ attributed to organics. We assess the shapes and strengths of these absorptions in the context of laboratory spectra of extraterrestrial organics and analogs.

Results. We find spectral evidence of aromatic and aliphatic $\mathrm{CH}$ bonds. The absorptions are broadly consistent in shape and depth with those associated with insoluble organic matter in meteorites. Given the thermal and space weathering environments on Bennu, it is likely that the organics have not been exposed for long enough to substantially decrease the $\mathrm{H} / \mathrm{C}$ and destroy all aliphatic molecules.
\end{abstract}

Key words. minor planets, asteroids: individual: (101955) Bennu - techniques: spectroscopic - planets and satellites: composition

\section{Introduction}

The Origins, Spectral Interpretation, Resource Identification, and Security-Regolith Explorer (OSIRIS-REx) mission detected carbon species, including organics and carbonates, on nearEarth asteroid (101955) Bennu (Kaplan et al. 2020; Simon et al. 2020a). These findings, based on the presence of an infrared absorption near $3.4 \mu \mathrm{m}$, indicate that the sample of Bennu's regolith that the OSIRIS-REx spacecraft will return to Earth in 2023 (Lauretta et al. 2021, 2017) is likely to contain carbonbearing material. The organic component may hold clues to the conditions of the early Solar System and the origins of life on Earth (e.g., Chyba et al. 1990).

Bennu has been spectrally linked to aqueously altered CI- and CM-type carbonaceous chondrites (Clark et al. 2011; Hamilton et al. 2019); these primitive carbon-rich meteorites are likely the closest analogs of Bennu currently available for laboratory studies. CI and CM chondrites contain 1 to $5 \mathrm{wt} . \%$ total carbon in organic and inorganic forms (Pearson et al. 2006; Alexander et al. 2012; Sephton 2002). The majority of the meteoritic carbon $(>70 \mathrm{wt} . \%)$ is hosted in insoluble organic matter (IOM): an acid-insoluble kerogen-like macromolecule that is structurally complex, with variable isotopic and elemental compositions (Cody \& Alexander 2005; Alexander et al. 2017). The soluble organic matter (SOM) represents a much smaller fraction (up to $0.1 \%$ ) of the total carbon, with the remainder of carbon either unaccounted for with current techniques or contained in inorganic forms (e.g., carbonates and nanodiamonds). Meteoritic SOM can be highly complex, with a variety of different compound classes, such as amino acids, carboxylic acids, hydroxy acids, amines, alcohols, aldehydes, ketones, $\mathrm{N}$-heterocycles, polyols, aliphatic and aromatic hydrocarbons, and sugars (e.g., Glavin et al. 2018).

Most of the meteoritic organic matter likely originated in the interstellar medium or in the colder regions of the protoplanetary disk before being incorporated into the earliest Solar 
System bodies. Such bodies include the parent asteroids of the carbonaceous chondrites and of Bennu, which is the rubble-pile descendant of a larger body that was catastrophically disrupted (e.g., Barnouin et al. 2019; Michel et al. 2020). Differences in the organic abundances, distributions, and isotopic and elemental compositions within and between carbonaceous chondrite groups have been attributed to nebular processes, thermal and/or aqueous alteration of the parent body (e.g., Herd et al. 2011; Alexander et al. 2007; Glavin et al. 2010), and surface modification, such as space weathering (e.g., Thompson et al. 2020).

\section{Observations and methods}

The OSIRIS-REx Visible and InfraRed Spectrometer (OVIRS; Reuter et al. 2018) has a circular, 4 mrad field of view. It collected tens of thousands of spectra of Bennu with wavelengths from 0.4 to $4.3 \mu \mathrm{m}$. In the wavelength region near $3.4 \mu \mathrm{m}(3.2$ to $3.6 \mu \mathrm{m})$, there are at least four absorption characteristic of organic carbon, resulting from the symmetric and asymmetric stretching modes of methyl $\left(-\mathrm{CH}_{3}\right)$ and methylene $\left(-\mathrm{CH}_{2}\right)$ groups (i.e., aliphatic CH; e.g., Allen \& Wickramasinghe 1981; Pendleton 1995).

We analyzed spectra collected during the sample site reconnaissance (Recon A) phase of the OSIRIS-REx mission in October 2019 (Lauretta et al. 2021, 2017). The Recon A data set in this study comprises 15585 photometrically corrected OVIRS spectra that cover $10 \%$ of the surface; it focuses on regions of interest, including the Nightingale site (Hokioi crater), where OSIRIS-REx collected its sample (see Appendix A for more observation details and coverage map). We used these data, rather than the global-coverage data set at $20 \mathrm{~m}$ per footprint (Simon et al. 2020a), because the spatial resolution (4-5 m cross-track and $7-10 \mathrm{~m}$ along-track) is optimized to isolate organic-rich regions of the surface.

We used laboratory spectra to directly compare the OVIRS spectra from 2.9 to $3.6 \mu \mathrm{m}$ with well-studied organic endmembers. Laboratory spectra included in our analyses are of (i) IOM extracted from carbonaceous chondrites; (ii) material captured from comet $81 \mathrm{P} /$ Wild 2 by the Stardust spacecraft, which may have been modified by the capture process, though the spectrum resembles spectra of primitive interplanetary dust particles (Keller et al. 2006); (iii) a laboratory-made, irradiated ice tholin, which was created to approximate cometary organic matter (Khare et al. 1993); and (iv) terrestrial asphaltite (Moroz et al. 1998) (Figs. 1c, 2, and Table A.1). These laboratory endmembers were chosen as the most likely organic components to be observed at the macroscale on Bennu based on meteorite analyses and previous observations (Simon et al. 2020a). We also included a set of ammonium salt spectra with our laboratory endmembers to distinguish nitrogen-bearing phases, which may be present on comets and some asteroids and have absorptions in the wavelength range $(2.9-3.6 \mu \mathrm{m})$ studied here (Poch et al. 2020).

We used linear least-squares and Kolmogorov-Smirnov goodness-of-fit methods to determine the closest matches between OVIRS spectra and laboratory spectra with respect to spectral shape (see Appendix A). The same least-squares comparison method was previously used to identify carbonate-rich spots on Bennu by identifying $3.4 \mu \mathrm{m}$ features with a doublet spectral shape and band positions indicative of carbonates rather than organics; we find that least-squares fits with $\chi^{2}$ values $<2$ provide credible matches to the OVIRS data (Kaplan et al. 2020). The Kolmogorov-Smirnov method was subsequently used to confirm these results (Ferrone et al. 2021, and see our Appendix A).

Finally, we measured the strength of the $\mathrm{CH}$ absorption feature from the continuum at $3.42 \mu \mathrm{m}$ (i.e., band depth at $3.42 \mu \mathrm{m}$; Clark \& Roush 1984) as another method for assessing organic composition $(\mathrm{H} / \mathrm{C})$ and concentration $(\mathrm{C}$ wt.\%). The band depth at $3.42 \mu \mathrm{m}$ has been linked to the composition and concentration of organic matter in sedimentary rocks and meteorites (e.g., Herron et al. 2014; Kaplan \& Milliken 2018). For IOM extracted from carbonaceous chondrites, band depth is positively correlated with the hydrogen-to-carbon ratio $(\mathrm{H} / \mathrm{C}$; Kaplan et al. 2019). If there is not enough hydrogen in the organics (i.e., $\mathrm{H} / \mathrm{C}<0.3$ ), there will be no absorption at $3.42 \mu \mathrm{m}$. For IOM in bulk rock (i.e., meteorites), the absolute concentration is also important: $>1 \mathrm{wt}$.\% $\mathrm{C}$ is needed to observe an absorption at $3.42 \mu \mathrm{m}$ (Kaplan et al. 2019). Most CMs and CIs contain $1-3$ wt. $\% \mathrm{C}$ and have bulk $\mathrm{H} / \mathrm{C}$ ratios in the $\mathrm{IOM}>0.5$ (Alexander et al. 2007).

\section{Results}

\subsection{Spectral features}

Most OVIRS spectra appear to be a mixture of organic and carbonate material, which is not suitable for our analysis. In total, only 237 of the 15585 spectra analyzed are well fit by the organic laboratory spectra described in Sect. $2\left(\chi^{2}<2\right.$; see Appendix A). We refer to these hereafter as organic-rich spectra because they have absorption band positions and widths that match laboratory organic spectra (Fig. 1). These organicrich spectra are distributed across the asteroid surface (i.e., are not concentrated in any given region), suggesting a wide spatial distribution of organics (Ferrone et al. 2021). The spectral features themselves are not homogenous and have varying absorption strengths and shapes (Simon et al. 2020a).

The organic-rich spectra typically have a strong absorption at $3.42 \mu \mathrm{m}$, which is consistent with the position of the symmetric $-\mathrm{CH}_{2}$ stretch (Fig. 1b). The entire absorption spans from 3.2 to $3.5 \mu \mathrm{m}$, covering the full set of aliphatic $\mathrm{CH}$ stretches. The surface-averaged OVIRS spectrum (Figs. 1a,b, red spectrum) has a minimum at $3.47 \mu \mathrm{m}$ that likely reflects the contribution from carbonates. The asymmetric $\mathrm{CH}$ stretches (3.38 or $3.42 \mu \mathrm{m}$ ) are typically stronger than their longer wavelength (lower wavenumber) symmetric counterparts, so spectra dominated by aliphatic organics should not have the strongest feature at $3.47 \mu \mathrm{m}$. Although there does appear to be some structure within this region, the most identified absorptions $(3.38,3.42$, 3.47 , and $3.5 \mu \mathrm{m}$ ) are not as well resolved in the OVIRS spectra as they are in some laboratory spectra (e.g., Cold Bokkeveld $\mathrm{CM}$ chondrite; Fig. 1). The signal-to-noise ratio $(\mathrm{S} / \mathrm{N})$ and spectral resolution for the OVIRS data in this region are both lower than that of the laboratory spectra, which likely accounts for the inability to resolve the individual organic absorptions (see our Fig. A.2 and Appendix A as well as Reuter et al. 2018); laboratory spectra with noise added to achieve S/N of the OVIRS spectra are shown in Figs. 1 and 2 (see Appendix A).

Some of the OVIRS organic-rich spectra appear to have a "shoulder" or weak absorption near $3.3 \mu \mathrm{m}$ that is consistent with aromatic $\mathrm{CH}$ stretching (Figs. $1 \mathrm{~b}$ and A.3). Although this spectral feature is common in some pure, laboratory organics, it is uncommon in bulk meteorite spectra owing to stronger, overlapping hydration features and a low absorption cross section (Dartois et al. 2004; Kaplan et al. 2019). A strong absorption band at $3.3 \mu \mathrm{m}$ has, however, been observed on comet 

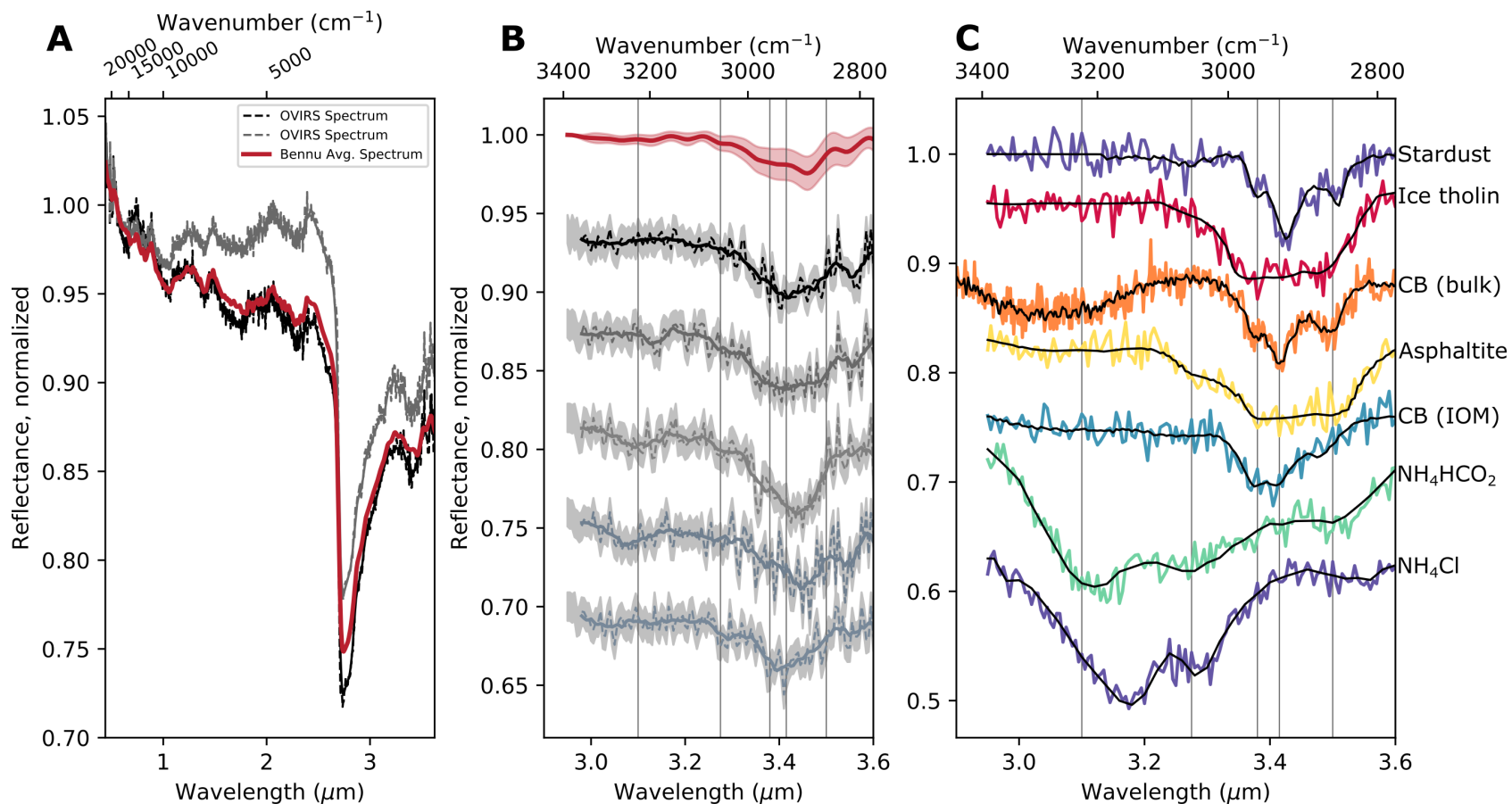

Fig. 1. Evidence of organics on Bennu in spectral features near $3.4 \mu \mathrm{m}$. (a) Average OVIRS spectrum of Bennu (red) and two OVIRS spectra of Bennu with spectral features near $3.4 \mu \mathrm{m}$. (b) Close-up of the continuum-removed region around $3.4 \mu \mathrm{m}$, offset, with wavelengths of $\mathrm{C}-\mathrm{H}$ absorptions indicated by vertical gray lines. The top three spectra are the same as those shown in panel a. The shaded region indicates uncertainties, and the vertical lines at $3.10,3.275,3.38,3.42$, and $3.50 \mu \mathrm{m}$ indicate positions of possible absorption features. (c) Laboratory spectra near $3.4 \mu \mathrm{m}$ for comparison. Black lines are the spectra, and colors are spectra with noise added to simulate OVIRS noise. "CB" stands for "Cold Bokkeveld", "bulk" refers to the whole meteorite, and "IOM" refers to the extracted insoluble organic component.

67P/Churyumov-Gerasimenko (67P) by the Rosetta mission, suggesting the presence of polycyclic aromatic hydrocarbons with free aromatic $\mathrm{CH}$ stretching (Raponi et al. 2020). An alternative explanation of the $3.3 \mu \mathrm{m}$ band on 67P, along with an absorption at $3.1 \mu \mathrm{m}$, is $\mathrm{N}-\mathrm{H}$ vibration modes from the presence of ammonia salts (Poch et al. 2020). Some OVIRS spectra of Bennu do appear to have a weak feature at $3.1 \mu \mathrm{m}$ that is consistent with some NH-bearing phases (Figs. 1a, 2c, and A.3)

Outside of the 2.9 to $3.6 \mu \mathrm{m}$ wavelength region, the organicrich spectra are broadly similar to the average visible and nearinfrared spectrum of Bennu, which has a hydration feature at $2.74 \mu \mathrm{m}$, suggesting that the surface composition is dominated by Mg-rich phyllosilicates (Hamilton et al. 2019) (Fig. 1a). There is also a series of minor absorption features at shorter wavelengths $(<2.5 \mu \mathrm{m})$ that are related to the hydrated phyllosilicates and iron oxides (Simon et al. 2020b). We do not find a correlation between any phyllosilicate spectral features and organic features in the Recon A spectra, which is consistent with the coarser-scale global OVIRS observations (Simon et al. 2020a).

\subsection{Organic $H$ and $C$ abundances}

In terms of overall spectral shape, IOM extracted from the carbonaceous chondrite meteorites are the best fit to all organicrich OVIRS spectra (Fig. 2). We find some examples (Fig. 2b) where there may be additional absorption features not seen in the IOM spectra, but the feature at $3.2-3.6 \mu \mathrm{m}$ is still the dominant absorption, and IOM provides the best spectral match of any laboratory endmember. The 18 IOM laboratory spectra used as laboratory spectral endmembers have similar spectral shapes, with small differences in methyl and methylene absorption strengths ( $3.38 \mu \mathrm{m}$ vs. $3.42 \mu \mathrm{m}$ band depth) that result from a different aliphatic chain length (e.g., Orthous-Daunay et al. 2013; Kebukawa et al. 2011). Using the linear least-squares and Kolmogorov-Smirnov fitting techniques, we can distinguish IOM spectral shapes from other organics and carbonates in data with $\mathrm{S} / \mathrm{N}$ of the OVIRS data with high confidence (Table A.2), but differentiating between IOM from individual meteorites is more difficult (Table A.3). Therefore, we group spectral fits as generally IOM-like.

The carbonaceous chondrites and IOM provide constraints on the elemental composition of Bennu's organics. The presence of an aliphatic spectral feature in IOM indicates that the H/C ratio is greater than 0.3 (Kaplan et al. 2019), suggesting that the minimum $\mathrm{H} / \mathrm{C}$ on Bennu is 0.3. Using band depth at $3.42 \mu \mathrm{m}$ to estimate the $\mathrm{H} / \mathrm{C}$ from the spectrum, we find $\mathrm{H} / \mathrm{C}$ values ranging from 0.4 to 0.6 for the organic-rich spectra of Bennu identified in this study (Fig. 3a). Given that the contribution from carbonate in the spectral region near $3.4 \mu \mathrm{m}$ is unknown (Kaplan et al. 2020) and given the contribution of organic abundance to the $3.42 \mu \mathrm{m}$ band depth (Kaplan et al. 2019), these values are upper limits. The high end of this range is typical of the CM and CI chondrites (Fig. 3a), which are Bennu's closest analogs (Hamilton et al. 2019, 2021).

Estimates of organic abundances on Bennu are greatly complicated by the presence of carbonates, which have overlapping spectral features at $3.4 \mu \mathrm{m}$ (Kaplan et al. 2020). In addition, organic spectral models for assessing abundance can be strongly affected by organic composition, making it difficult to determine exact abundances, as has been shown for Ceres (Kaplan et al. 2018). That said, the $3.42 \mu \mathrm{m}$ band depths observed in the OVIRS spectra studied here are consistent with carbonaceous chondrites that contain 1-2.5 wt.\% C (Fig. 3b), suggesting that similar concentrations of organics could be present on Bennu. 

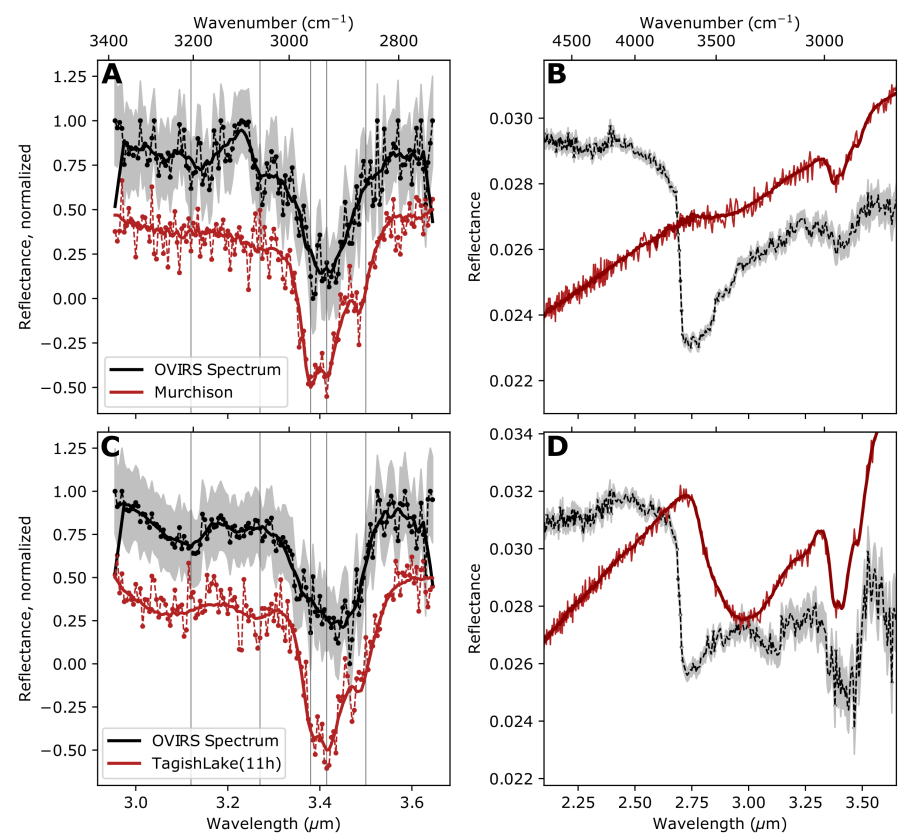

Fig. 2. Laboratory spectral matches for organic spectral shapes observed on Bennu. The most common spectral shapes in the Bennu spectra that we analyzed are well matched with spectra of meteorite IOM. $(a)$ and (c) Best fits in the 3.1 to $3.6 \mu \mathrm{m}$ region between the OVIRS spectra (black) and the IOM spectra (red, solid), with noised added to simulate OVIRS noise (red, dashed); the spectra are normalized from 0 to 1 and offset for clarity. The shaded region indicates uncertainties, and vertical lines at $3.10,3.275,3.38,3.42$, and $3.50 \mu \mathrm{m}$ indicate positions of possible absorption features. $(b)$ and $(d)$ OVIRS and IOM spectra from 2.1 to $3.6 \mu \mathrm{m}$ shown without normalization or continuum removal; IOM spectra have been scaled.

The organic-rich regions of Ceres, on the other hand, have a stronger absorption feature at $3.4 \mu \mathrm{m}$ than any carbonaceous chondrite. This strong feature has been used as evidence of an endogenous mechanism for further concentrating organic matter beyond what was originally incorporated into the body (De Sanctis et al. 2019, 2017; Kaplan et al. 2018; Marchi et al. 2019), a mechanism that is neither likely in Bennu's case nor needed to explain the spectra.

The $3.42 \mu \mathrm{m}$ band depth reflects the combination of organic composition (e.g., H/C) and abundance (e.g., C wt.\%) in bulk meteorites (Fig. 3c). If we assume there is $2.5 \mathrm{wt} . \% \mathrm{C}$ on Bennu, then the range in $3.42 \mu \mathrm{m}$ band depth for the organic-rich spectra is consistent with a $\mathrm{H} / \mathrm{C}$ of 0.3 to 0.6 ; smaller $\mathrm{C}$ abundances would require high $\mathrm{H} / \mathrm{C}$ values. To untangle these values will require additional laboratory measurements of the returned samples. However, the ranges of $\mathrm{H}$ and $\mathrm{C}$ values suggested by Bennu's $3.4 \mu \mathrm{m}$ absorption feature are broadly consistent with the carbonaceous chondrites and fall in the same range as available CM chondrite spectra.

Absorption features due to $\mathrm{C}-\mathrm{C}, \mathrm{C}=\mathrm{C}$, and $\mathrm{C}=\mathrm{O}$ bonds result from aromatic structures, ketones, and carboxyls, which are prominent in meteorite IOM; however, they are absent in the wavelength range covered by OVIRS spectra $(0.4$ to $4.3 \mu \mathrm{m})$ Therefore, we expect there to be an additional, unknown amount of carbon in aromatic structures, reduced compounds (nanodiamond, graphite, and carbides), or amorphous material that is not detectable with OVIRS and cannot be quantified from our observations but will be determined from the returned sample.

\section{Discussion}

The spectral features from 2.9 to $3.6 \mu \mathrm{m}$ observed on Bennu allow us to compare this asteroid to other extraterrestrial settings. The OVIRS data are comparable to the spectra seen in the diffuse interstellar medium (Allen \& Wickramasinghe 1981; Sandford et al. 1991), comet 67P (Raponi et al. 2020), and multiple large main-belt asteroids (Simon et al. 2020a). We find that carbonaceous chondrite IOM is the closest laboratory ana$\log$ to Bennu's organics based on the available spectral data at the wavelengths covered by the OVIRS instrument. This finding strengthens the previously described connection between Bennu and carbonaceous chondrite meteorites (Clark et al. 2011; Hamilton et al. 2019, 2021). The macroscale heterogeneity of the organic-rich spectra of Bennu has not been observed elsewhere in the Solar System (potentially owing to a lack of spacecraft data in most cases) but is mirrored in the large heterogeneity seen at small scales in meteorite organics (e.g., Alexander et al. 2017).

The organics on Bennu may reflect heterogenous aqueous alteration conditions. Although aqueous alteration has been reported to decrease $\mathrm{H} / \mathrm{C}$ (Herd et al. 2011), the modification of organics is primarily controlled by heating (Alexander et al. 2014; Quirico et al. 2018), and the variation in H/C and C wt.\% estimated for organic-rich OVIRS spectra may be the result of variable heating (Fig. 3). Typically, higher $\mathrm{H} / \mathrm{C}$ values are also associated with higher $\mathrm{N} / \mathrm{C}, \mathrm{O} / \mathrm{C}$, and bulk $\mathrm{C}$ (Alexander et al. 2007). Though spectra with a feature near $3.1 \mu \mathrm{m}$ may indicate the presence or increased abundance of nitrogen-bearing phases, the absorption is weak, and more work is needed to understand the possibility of these phases on Bennu.

However, outside of the $3.4 \mu \mathrm{m}$ region, Bennu is spectrally similar to $\mathrm{CI}$ and $\mathrm{CM}$ carbonaceous chondrites of petrographic subtypes $<2.4$, based on the Rubin et al. (2007) scale, including some that are mildly heated (Hamilton et al. 2019, 2021; Kaplan et al. 2020). Other indications of aqueous alteration, such as the $2.7 \mu \mathrm{m}$ hydration band depth, do not correspond to the organic variation.

Bennu's surface has been exposed to high temperatures during its 1.75 million years in near-Earth space (Ballouz et al. 2020). It has also been exposed to weathering in the space environment for as many as 50 million to 1 billion years, based on maximum crater retention ages (Walsh et al. 2019; Bierhaus et al., in prep). Temperature and solar wind irradiation breaks down aliphatic organics by removing hydrogen (Kebukawa et al. 2010; Laczniak et al. 2021), weakening or erasing the aliphatic absorption feature at $3.4 \mu \mathrm{m}$. Kebukawa et al. (2010) used Murchison IOM (one of the laboratory organic analogs used in this study) to determine that aliphatic $\mathrm{CH}$ is lost within 200 years at $100^{\circ} \mathrm{C}$ $(373 \mathrm{~K})$. Bennu's maximum surface temperatures exceed this temperature for multiple hours each day at perihelion for much of the surface (Rozitis et al. 2020), which would lead to the destruction of aliphatic organics over Bennu's near-Earth lifetime. However, small-scale surface roughness allows for some regions (meter-scale or smaller) to continuously maintain the maximum temperatures, $<0^{\circ} \mathrm{C}(273 \mathrm{~K})$, necessary to extend the aliphatic organic lifetime to 100 million years (Rozitis et al. 2020; Kebukawa et al. 2010), and small-scale surface temperature heterogeneity could lead to the observed differences in organic band depth and spectral shape (Figs. 2 and 3).

Laboratory experiments have also shown diverging effects on the spectral trends and organic chemistry resulting from various constituent space weathering processes. Experiments simulating the micrometeoroid bombardment of carbonaceous 

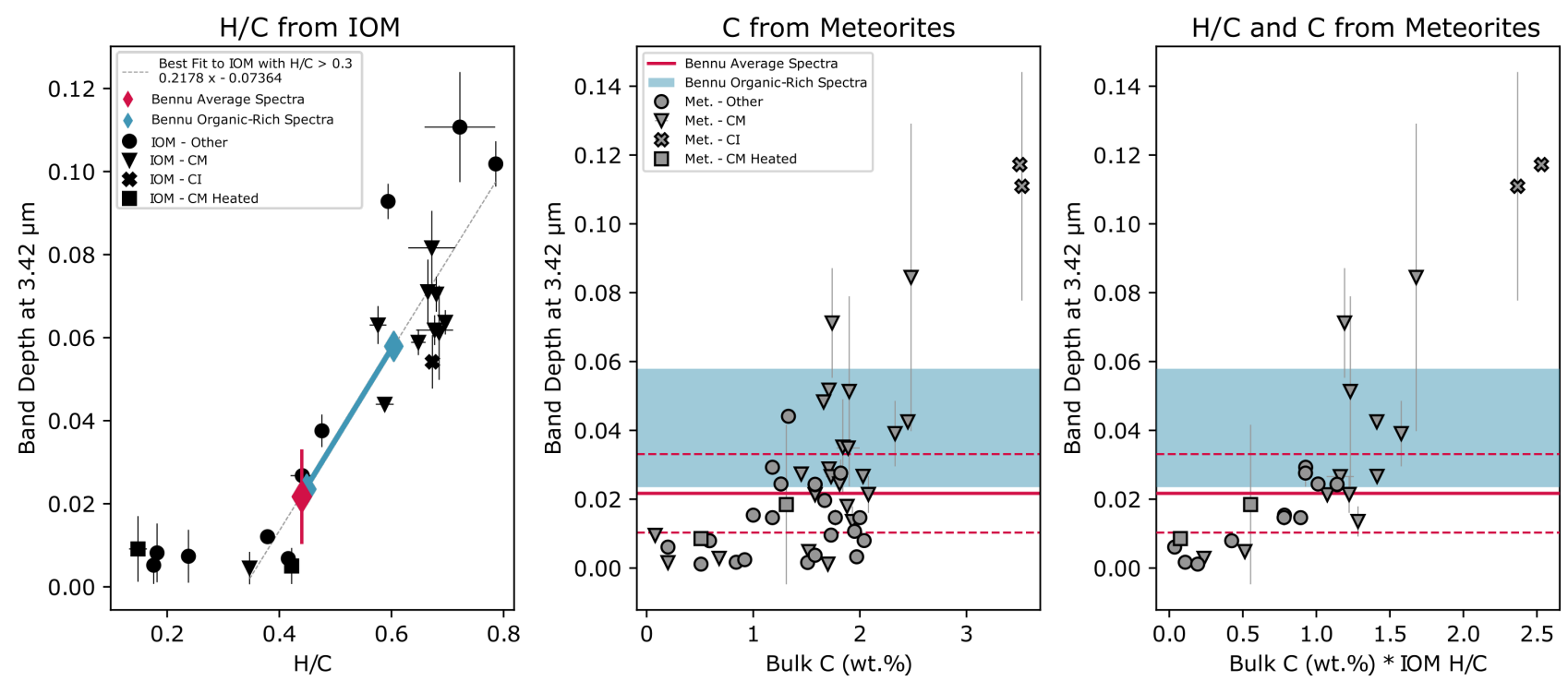

Fig. 3. Estimated H/C. (a) Carbonaceous chondrite IOM band depth at $3.42 \mu \mathrm{m}$, plotted against H/C (atom.) from Alexander et al. (2007); "other" refers to IOM from CV, CO, CR, and ungrouped carbonaceous chondrites. The $3.42 \mu \mathrm{m}$ band depths from OVIRS Recon A average (red diamond) and organic-rich spectra (blue diamonds) are plotted on the IOM trend to estimate the upper limit on H/C of 0.6 for Bennu. (b) Carbonaceous chondrite (i.e., bulk meteorite) band depth at $3.42 \mu \mathrm{m}$ plotted against wt.\% $\mathrm{C}$ for those meteorites; the symbology is the same as in panel a but for bulk meteorites as opposed to IOM (gray triangle, CM-type; gray cross, CI-type; gray square, heated CM-type; gray circle, other carbonaceous chondrite IOM, including CV, CO, CR, and ungrouped). The band depth at $3.42 \mu \mathrm{m}$ is also shown for OVIRS organic-rich (blue shaded region) and Bennu global average spectra (solid red line; standard deviation, dashed red lines). The Bennu organic-rich spectra have similar band depths to carbonaceous chondrites containing 1-2.5 wt.\% C. (c) Combination of wt. $\% \mathrm{C}$ and $\mathrm{H} / \mathrm{C}$ of IOM, the best predictor of $3.42 \mu \mathrm{m}$ band depth in the meteorites.

chondrites have shown an increase in detectable organics correlated with darkening and mild bluing slopes (Thompson et al. 2020). In contrast, studies simulating solar wind irradiation have shown reddening of the spectral slope correlated with a mixed efficiency for organic species destruction. Irradiation with $1 \mathrm{keV}$ $\mathrm{H}+$ ions resulted in only limited, if any, destruction of organics and mild spectral reddening, whereas $4 \mathrm{keV} \mathrm{He}+$ irradiation resulted in strong reddening and a reduction in detectable organics by up to $40 \%$ over 10000 -year timescales (Laczniak et al. 2021). Thus, the space weathering of organics may result in competitive spectral trends, depending on the dominant constituent processes, initial composition, and/or the timescales of surface exposure.

The potential for the rapid breakdown of aliphatic organics would indicate that where these compounds are observed on Bennu, the surface has been exposed for a relatively short period of time. Spectrophotometric studies suggest that Bennu's small craters may be less than tens of thousands of years old (DellaGiustina et al. 2020). In addition, evidence of particle ejection and re-impact (Lauretta et al. 2019) and thermally driven fracturing (Molaro et al. 2020) on Bennu indicates that ongoing surface processes are continually exposing fresh material at the surface.

\section{Conclusions}

The detection of organic matter on asteroid Bennu using the OVIRS instrument on board the OSIRIS-REx spacecraft provides a preview of the composition of the sample that will be returned to Earth in 2023. Spectral features near $3.4 \mu \mathrm{m}$, including a strong absorption minimum between 3.38 and $3.42 \mu \mathrm{m}$ and a possible shoulder at $3.3 \mu \mathrm{m}$, are indicative of aliphatic and aromatic $\mathrm{CH}$ with a maximum $\mathrm{H} / \mathrm{C}$ of 0.6 and a minimum $\mathrm{H} / \mathrm{C}$ of 0.3 . The overall spectral shape in the OVIRS data is most comparable to meteorite IOM, as opposed to other organic analogs. Elevated temperatures and space weathering should quickly alter these absorption features, so the strongest organic spectral features likely indicate the organic material relatively recently exposed by ongoing mass shedding and impact processes and/or material that has been shielded from relatively high temperatures. The sample of Bennu's regolith returned by OSIRIS-REx will tell us about the organic chemistry on Bennu beyond the $\mathrm{CH}$-bearing material, particularly the detailed composition and structure of the IOM as well as details about the soluble organic diversity and composition.

Acknowledgements. We are grateful to the entire OSIRIS-REx Team for making the encounter with Bennu possible. This material is based upon work supported by NASA under Contract NNM10AA11C issued through the New Frontiers Program. OVIRS spectral data from Recon A are available via the Planetary Data System at https://sbn.psi.edu/pds/resource/orex/ovirs. html (Reuter et al. 2019). Data are delivered to the PDS according to the schedule in the OSIRIS-REx Data Management Plan, available in the OSIRISREx mission bundle at https://sbnarchive.psi.edu/pds4/orex/orex. mission/document/.

\section{References}

Alexander, C. M. O., Fogel, M., Yabuta, H., \& Cody, G. 2007, Geochim. Cosmochim. Acta, 71, 4380

Alexander, C. M. O., Bowden, R., Fogel, M. L., et al. 2012, Science, 337, 721

Alexander, C. M. O., Cody, G. D., Kebukawa, Y., et al. 2014, Meteor. Planet. Sci., 49, 503

Alexander, C., Cody, G., De Gregorio, B., Nittler, L., \& Stroud, R. 2017, Chem. Erde - Geochem., 77, 227

Allen, D. A., \& Wickramasinghe, D. T. 1981, Nature, 294, 239

Ballouz, R.-L., Walsh, K. J., Barnouin, O. S., et al. 2020, Nature, 587, 205

Barnouin, O. S., Daly, M. G., Palmer, E. E., et al. 2019, Nat. Geosci., 12, 247

Chyba, C., Thomas, P., Brookshaw, L., \& Sagan, C. 1990, Science, 249, 366

Clark, R. N., \& Roush, T. L. 1984, J. Geophys. Res., 89, 6329

Clark, B. E., Binzel, R. P., Howell, E. S., et al. 2011, Icarus, 216, 462

Cody, G. D., \& Alexander, C. M. 2005, Geochim. Cosmochim. Acta, 69, 1085

Dartois, E., Muñoz Caro, G. M., Deboffle, D., \& d'Hendecourt, L. 2004, A\&A, 423, L33 
DellaGiustina, D. N., Burke, K. N., Walsh, K. J., et al. 2020, Science, 370, eabc3660

De Sanctis, M. C., Ammannito, E., McSween, H. Y., et al. 2017, Science, 355, 719

De Sanctis, M. C., Vinogradoff, V., Raponi, A., et al. 2019, MNRAS, 482, 2407

Ferrone, S., Clark, B., Kaplan, H., et al. 2021, Icarus, 368, 114579

Glavin, D. P., Callahan, M. P., Dworkin, J. P., \& Elsila, J. E. 2010, Meteor. Planet. Sci., 45, 1948

Glavin, D. P., Alexander, C. M., Aponte, J. C., et al. 2018, Primitive Meteorites and Asteroids (Elsevier), 205

Hamilton, V. E., Simon, A. A., Christensen, P. R., et al. 2019, Nat. Astron., 3 332

Hamilton, V. E., Christensen, P. R., Kaplan, H. H., et al. 2021, A\&A, 650, A120

Herd, C. D. K., Blinova, A., Simkus, D. N., et al. 2011, Science, 332, 1304

Herron, M., Loan, M., Charsky, A., et al. 2014, Petrophysics, 55, 434

Kaplan, H. H., \& Milliken, R. E. 2018, Clays Clay Miner., 66, 173

Kaplan, H. H., Milliken, R. E., \& Alexander, C. M. O. 2018, Geophys. Res. Lett., 45,5274

Kaplan, H. H., Milliken, R. E., Alexander, C. M. O., \& Herd, C. D. K. 2019, Meteor. Planet. Sci., 54, 1051

Kaplan, H. H., Lauretta, D. S., Simon, A. A., et al. 2020, Science, 370, eabc3557

Kebukawa, Y., Nakashima, S., \& Zolensky, M. E. 2010, Meteor. Planet. Sci., 45 99

Kebukawa, Y., Alexander, C. M., \& Cody, G. D. 2011, Geochim. Cosmochim. Acta, 75, 3530

Keller, L. P., Bajt, S., Baratta, G. A., et al. 2006, Science, 314, 1728

Khare, B., Thompson, W., Cheng, L., et al. 1993, Icarus, 103, 290

Laczniak, D., Thompson, M., Christoffersen, R., et al. 2021, Icarus, 364, 114479

Lauretta, D. S., Balram-Knutson, S. S., Beshore, E., et al. 2017, Space Sci. Rev. 212,925

Lauretta, D. S., Hergenrother, C. W., Chesley, S. R., et al. 2019, Science, 366, eaay 3544

Lauretta, D. S., Enos, H. L., Polit, A. T., Roper, H. L., \& Wolner, C. W. V. 2021, in Sample Return Missions, ed. A. Longobardo (Elsevier)
Marchi, S., Raponi, A., Prettyman, T. H., et al. 2019, Nat. Astron., 3, 140

Michel, P., Ballouz, R.-L., Barnouin, O. S., et al. 2020, Nat. Commun., 11, 2655

Molaro, J. L., Hergenrother, C. W., Chesley, S. R., et al. 2020, J. Geophys. Res. Planets, 125, e2019JE006325

Moroz, L., Arnold, G., Korochantsev, A., \& Wäsch, R. 1998, Icarus, 134, 253

Orthous-Daunay, F.-R., Quirico, E., Beck, P., et al. 2013, Icarus, 223, 534

Pearson, V. K., Sephton, M. A., Franchi, I. A., Gibson, J. M., \& Gilmour, I. 2006 Meteor. Planet. Sci., 41, 1899

Pendleton, Y. J. 1995, Planet. Space Sci., 43, 1359

Poch, O., Istiqomah, I., Quirico, E., et al. 2020, Science, 367, eaaw7462

Quirico, E., Bonal, L., Beck, P., et al. 2018, Geochim. Cosmochim. Acta, 241, 17

Raponi, A., Ciarniello, M., Capaccioni, F., et al. 2020, Nat. Astron., 4, 500 Reuter, D. C., Simon, A. A., Hair, J., et al. 2018, Space Sci. Rev., 214, 54

Reuter, D. C., Simon, A. A., Lunsford, A., \& Lauretta, D. S. 2019, Origins, Spectral Interpretation, Resource Identification, Security, Regolith Explorer (OSIRIS-REx): Visible and InfraRed Spectrometer (OVIRS)

Bundle, NASA Planetary Data System, https://arcnav.psi.edu/urn: nasa:pds: orex.ovirs

Rozitis, B., Emery, J. P., Siegler, M. A., et al. 2020, J. Geophys. Res.: Planets, $125, \mathrm{e} 06282$

Rubin, A. E., Trigo-Rodríguez, J. M., Huber, H., \& Wasson, J. T. 2007, Geochim. Cosmochim. Acta, 71, 2361

Sandford, S. A., Allamandola, L. J., Tielens, A. G. G. M., et al. 1991, ApJ, 371, 607

Sephton, M. A. 2002, Nat. Prod. Rep., 19, 292

Simon, A., Reuter, D., Gorius, N., et al. 2018, Remote Sens., 10, 1486

Simon, A. A., Kaplan, H. H., Hamilton, V. E., et al. 2020a, Science, 370, eabc3522

Simon, A. A., Kaplan, H. H., Cloutis, E., et al. 2020b, A\&A, 644, A148

Thompson, M., Morris, R. V., Clemett, S. J., et al. 2020, Icarus, 346, 113775

Walsh, K. J., Jawin, E. R., Ballouz, R.-L., et al. 2019, Nat. Geosci., 12, 242

Zou, X.-D., Li, J.-Y., Clark, B. E., et al. 2021, Icarus, 358, 114183 


\section{Appendix A: Supplementary material}

\section{A.1. Additional information on the data set}

We used OVIRS spectra from the Recon A phase of the OSIRIS-REx mission, specifically those that were collected on four separate dates in October 2019 (Reuter et al. 2019). These observations covered the mission's primary sample collection site, Nightingale, and three other candidate sample sites (Lauretta et al. 2021), as well as a few other areas of interest that were observed by opportunity. The spacecraft scanned the surface from a $1 \mathrm{~km}$ range with varying phase angles; the scan patterns are shown in Fig. A.1. OVIRS data collected during Recon A have a spatial resolution of 4 to $5 \mathrm{~m}$ cross-track and 7 to $10 \mathrm{~m}$ along-track $\left(36 \mathrm{~m}^{2}\right)$.

\section{A.2. Methods: Calibration and data processing}

Full details of the calibration pipeline are described in Simon et al. (2018). Briefly, each OVIRS spectrum comprises five segments collected from separate linear variable filters, with overlapping wavelengths that are resampled to create a continuous spectrum from 0.39 to $4.3 \mu \mathrm{m}$ (Reuter et al. 2018). A thermal tail is subtracted from calibrated radiance (resampled) spectra, and the result is divided by solar flux to obtain reflectance (I/F; Simon et al. 2020a). Thermal fill-in can occur when thermal emission at high temperatures leads to thermal flux within the band, causing absorption features to appear weak. However, there is no indication that the $3.4 \mu \mathrm{m}$ feature in the OVIRS spectra collected during Recon A is affected by thermal fill-in (Simon et al. 2020a).

To remove the confounding effects of viewing geometry, we used the photometric model developed by Zou et al. (2021) to correct the OVIRS data to standard viewing geometry (incidence $30^{\circ}$, emission $0^{\circ}$, phase $30^{\circ}$ ). However, this photometric correction had an insignificant effect at the wavelengths of interest in this study (e.g., $2.8-3.6 \mu \mathrm{m}$ ), and analyses with and without this correction are very similar.

A combination of warm surface and instrument temperatures led to decreasing $\mathrm{S} / \mathrm{N}$ at the longest OVIRS wavelengths, including within the wavelength region of interest (Fig. A.2). A detailed discussion of the wavelength-dependent $\mathrm{S} / \mathrm{N}$ for these OVIRS observations and its influence on the spectral detection of organics or carbonates on Bennu using the $3.4 \mu \mathrm{m}$ region can be found in Kaplan et al. (2020) and Simon et al. (2020a). Briefly, the Bennu S/N for Recon A was found by calculating the fractional RMS of calibrator spectra and then inverting and scaling that $\mathrm{S} / \mathrm{N}$ value by the ratio of the average radiance of an internal source to a representative Bennu spectrum for each observation date.

\section{A.3. Methods: Organic spectral detection and analysis}

We followed methods outlined in Simon et al. (2020a) and Kaplan et al. (2020) to perform our organic spectral analysis. Specifically, we used a three-point quadratic polynomial to fit to each OVIRS spectrum at 2.9,3.2, and $3.6 \mu \mathrm{m}$ to approximate the spectral continuum. We divided out this continuum and performed further analyses on the continuum-removed data set. We calculated band depths by taking an average of three channels surrounding the wavelength of interest and calculated the depth relative to the continuum reflectance. A set of laboratory spectra was resampled to OVIRS channels and treated in the same way for the sake of direct comparison (Tables A.2 and A.3). These spectra are all previously published, and references can be found in the main text. To compare spectral shape rather than feature strength, we normalized each continuum-removed spectrum to have a maximum value of 1 and a minimum value of 0 (Fig. 2). All analyses were performed on unsmoothed spacecraft data and reflect the noise inherent in spacecraft data; Figs. 1 and 2 also show spectra smoothed to demonstrate the likely underlying shape.

We used two previously tested methods to separate organic $(\mathrm{CH})$ absorptions near $3.4 \mu \mathrm{m}$ from the carbonate $\left(\mathrm{CO}_{3}\right)$ absorptions that are found at similar wavelengths to obtain the organicrich group of spectra (Kaplan et al. 2020; Ferrone et al. 2021); both methods resulted in similar rates of organic and carbonate identification for the Recon A data set (Ferrone et al. 2021). The first method is linear least-squares fitting, in which we compared (unsmoothed) OVIRS spectra and laboratory spectra (at their native $\mathrm{S} / \mathrm{N}$ but resampled to OVIRS spectral resolution) on a channel-by-channel basis from 2.9 to $3.6 \mu \mathrm{m}$ to find the laboratory spectrum that best fits the shape of the organic features observed in our Recon A data set. We assessed the goodness of fit with a $\chi^{2}$ statistic (Kaplan et al. 2020, Fig. A.4). Recon A spectra that were fit by one of the organic laboratory spectra with a $\chi^{2}$ value $<2$ were designated as organic-rich for this study, which resulted in 237 used for further analyses (e.g., in Fig. 3). The full list of laboratory analog spectra used in this study is found in Tables A.1 and A.4.

The second organic detection method is an adaptation of the Kolmogorov-Smirnov goodness of fit test (Ferrone et al. 2021). We calculated the cumulative sum of OVIRS and laboratory spectra as a function of wavelength to find the maximum distance between the two curves (OVIRS and laboratory) at any wavelength (Fig. A.4). The maximum distance (k) is used as our measure of goodness of fit. In Ferrone et al. (2021), Recon A spectra that were fit by one of the organic laboratory spectra with a $\mathrm{k}$ value $<0.028$ fell into the top $10 \%$ of fits, $25 \%$ of which were best fit with organic laboratory spectra. We confirm with this method that the 237 organic-rich spectra obtained from linear-least-squares fitting were also positively identified with the Kolmogorov-Smirnov method.

We tested our spectral classification methods by finding the best fitting spectral matches to noise-added laboratory spectra (Figs. 1, 2, and A.4). To simulate OVIRS noise, we generated random noise profiles and added them to the laboratory spectra, targeting a $\mathrm{S} / \mathrm{N}$ of 50 , which is the nominal $\mathrm{S} / \mathrm{N}$ of the OVIRS spectra in the 3.1 to $3.6 \mu \mathrm{m}$ wavelength region and closely matches the Recon A S/N (Fig. A.2). We generated 10,000 random noise profiles for each of the laboratory spectra (e.g., we created 10,000 noise-added versions of the ice tholin spectrum). We performed the linear least-squares and Kolmogorov-Smirnov fitting to determine the noise-free laboratory spectrum that best matched each of the 10,000 noise-added spectra. We tested fitting with noise-free spectra that alternately include or exclude the subject spectrum. We find that the spectral matching correctly identifies the correct laboratory spectrum $>92 \%$ of the time if the noise-added laboratory spectrum is left out of the spectral group being used for fitting (i.e., a case where we simulate having similar organics to those on Bennu in our laboratory spectra group but not an exact match) and $>99 \%$ of the time if the noise-added laboratory spectrum is kept in the spectral fitting group (i.e., a case where we simulate having an exact match for Bennu organics in our laboratory spectra; Table A.2). 
Table A.1. Candidate analog organic materials.

\begin{tabular}{|c|c|c|c|}
\hline Name & Type & Source & Spectrum ID \\
\hline Cold Bokkeveld IOM & Meteorite IOM & Kaplan et al. 2019 & n.a. \\
\hline DOM08006 IOM & Meteorite IOM & Kaplan et al. 2019 & n.a. \\
\hline GRO95577 IOM & Meteorite IOM & Kaplan et al. 2019 & n.a. \\
\hline QUE97008 IOM & Meteorite IOM & Kaplan et al. 2019 & n.a. \\
\hline Tag_UA_11h IOM & Meteorite IOM & Kaplan et al. 2019 & n.a. \\
\hline Tagish_CCL IOM & Meteorite IOM & Kaplan et al. 2019 & n.a. \\
\hline Tagish_DM IOM & Meteorite IOM & Kaplan et al. 2019 & n.a. \\
\hline Tagish_Lake IOM & Meteorite IOM & Kaplan et al. 2019 & n.a. \\
\hline ALH83100 & Meteorite IOM & Kaplan et al. 2019 & n.a. \\
\hline ALH85013 IOM & Meteorite IOM & Kaplan et al. 2019 & n.a. \\
\hline Bells IOM & Meteorite IOM & Kaplan et al. 2019 & n.a. \\
\hline GRO95566 IOM & Meteorite IOM & Kaplan et al. 2019 & n.a. \\
\hline LEW85311 IOM & Meteorite IOM & Kaplan et al. 2019 & n.a. \\
\hline MET01070 IOM & Meteorite IOM & Kaplan et al. 2019 & n.a. \\
\hline Mighei IOM & Meteorite IOM & Kaplan et al. 2019 & n.a. \\
\hline Murchison IOM & Meteorite IOM & Kaplan et al. 2019 & n.a. \\
\hline Orgueil IOM & Meteorite IOM & Kaplan et al. 2019 & n.a. \\
\hline Y86720 IOM & Meteorite IOM & Kaplan et al. 2019 & n.a. \\
\hline K-Oxalate & Oxalate & Applin et al. 2019 & n.a. \\
\hline Na-Oxalate & Oxalate & Applin et al. 2019 & n.a. \\
\hline Ca-Oxalate & Oxalate & Applin et al. 2019 & n.a. \\
\hline $\mathrm{Fe}$-Oxalate & Oxalate & Applin et al. 2019 & n.a. \\
\hline Mg-Oxalate & Oxalate & Applin et al. 2019 & n.a. \\
\hline NH4 citrate dibasic (with pyrrhotite) & Ammonium salt & Poch et al. 2020 & NH4_citrate_dibasic_23wtpc_pyrrhotite \\
\hline $\mathrm{NH} 4 \mathrm{Cl}$ (with pyrrhotite) & Ammonium salt & Poch et al. 2020 & NH4Cl_9wtpc_pyrrhotite \\
\hline NH4HCO2 (with pyrrhotite) & Ammonium salt & Poch et al. 2020 & NH4HCO2_17wtpc_pyrrhotite \\
\hline NH4SO4 (with pyrrhotite) & Ammonium salt & Poch et al. 2020 & NH4SO4_9wtpc_pyrrhotite \\
\hline NH4SO4 (with pyrrhotite) & Ammonium salt & Poch et al. 2020 & NH4SO4_23wtpc_pyrrhotite \\
\hline Asphaltite & Complex OM & Moroz et al. 1998 & n.a. \\
\hline Ice Tholin & Complex OM & Khare et al. 1993 & n.a. \\
\hline Tyrosine & Amino acid & RELAB Spectral Library & CH-JLB-mach06 \\
\hline Stardust & Complex OM & Keller et al. 2006 & n.a. \\
\hline
\end{tabular}

Notes. Samples of candidate analog organic materials used in this study, including their source and spectrum identifier where available.

Table A.2. Spectral fitting methods applied to organic analog spectra.

\begin{tabular}{|l|l|l|l|l|}
\hline & \multicolumn{5}{|c|}{ \% Positive Identifications for 10,000 Fits } \\
\hline & \multicolumn{3}{|c|}{ All Lab Endmembers Included } & \multicolumn{2}{c|}{ Endmember to be Fit Left Out } \\
\hline Type & Group ID - Linear & Group ID - K-S & Group ID - Linear & Group ID - K-S \\
\hline Organics & 99.94 & 92.21 & 88.78 \\
\hline Meteorite IOM & 99.75 & 99.31 & n.a. & n.a. \\
\hline Oxalate & 99.46 & 99.34 & n.a. & n.a. \\
\hline Ammonium Salt & 95.88 & 84.76 & n.a. & n.a. \\
\hline Asphaltite & 99.53 & 87.30 & n.a. & n.a. \\
\hline Ice Tholin & 99.98 & 99.29 & n.a. & n.a. \\
\hline Stardust & 95.33 & 96.39 & 95.75 & 93.25 \\
\hline Carbonates & 96.94 & 94.10 & 91.62 & 89.14 \\
\hline Calcite & 97.13 & 94.04 & 88.35 & 86.61 \\
\hline Dolomite & 94.92 & \multicolumn{2}{|l}{} \\
\hline Magnesite & \multicolumn{5}{|l|}{} \\
\hline
\end{tabular}

Notes. Linear least-squares (linear) and Kolmogorov-Smirnov (K-S) fitting methods were applied to laboratory endmembers with 10,000 randomly generated noise profiles simulating OVIRS noise. We fit the noise-added laboratory spectra using the noise-free laboratory endmembers listed in Tables A.1 and A.4, either including the spectrum being fit (all laboratory endmembers included) or leaving out the spectrum being fit (endmembers left out). 
Table A.3. Spectral fitting methods applied to IOM spectra.

\begin{tabular}{|l|l|l|l|l|}
\hline \multicolumn{7}{|}{$\%$ Positive Identifications for 10,000 Fits } \\
\hline IOM Name & ID Individual - Linear & ID Individual - K-S & ID as IOM - Linear & ID as IOM - K-S \\
\hline Cold Bokkeveld & 92.19 & 51.65 & 100 & 100 \\
\hline DOM08006 & 93.7 & 94.35 & 100 & 99.99 \\
\hline GRO95577 & 41.88 & 27.47 & 100 & 99.99 \\
\hline QUE97008 & 100 & 99.86 & 100 & 99.86 \\
\hline Tag_UA_11h & 92.06 & 68.85 & 100 & 99.97 \\
\hline Tagish_CCL & 48.08 & 23.52 & 100 & 99.99 \\
\hline Tagish_DM & 99.4 & 95.32 & 100 & 99.98 \\
\hline Tagish_Lake & 94.38 & 95.72 & 100 & 100 \\
\hline ALH83100 & 45.82 & 21.19 & 100 & 99.98 \\
\hline ALH85013 & 46.14 & 31.01 & 100 & 100 \\
\hline Bells & 59.86 & 22.14 & 100 & 100 \\
\hline GRO95566 & 30.77 & 19.72 & 100 & 99.99 \\
\hline LEW85311 & 38.49 & 31.42 & 100 & 100 \\
\hline MET01070 & 36.32 & 35.63 & 100 & 99.99 \\
\hline Mighei & 45.91 & 40.21 & 100 & 99.94 \\
\hline Murchison & 42.2 & 23.05 & 100 & 99.99 \\
\hline Orgueil & 53.87 & 28.43 & 100 & 100 \\
\hline Y86720 & 95.42 & 99.32 & 95.42 & 99.32 \\
\hline
\end{tabular}

Notes. Results of our two spectral fitting methods, linear least-squares (linear) and Kolmogorov-Smirnov (K-S), when applied to IOM spectra with 10,000 randomly generated noise profiles simulating OVIRS noise. "ID individual" refers to cases in which the noise-added laboratory spectrum is best fit with its noise-free counterpart (i.e., an exact match); "ID as IOM" refers to cases where the noise-added laboratory spectrum is best fit with another IOM spectrum (as opposed to a different laboratory endmember, e.g., a calcite), which is true nearly $100 \%$ of the time.

Table A.4. Candidate analog carbonate materials.

\begin{tabular}{|c|c|c|c|}
\hline Name & Type & Source & Spectrum ID \\
\hline CAL110 <45 um; Calcite & Calcite & RELAB Spectral Library & CA-EAC-010_M1CA10 \\
\hline CRB109 <45 um; Calcite & Calcite & RELAB Spectral Library & CB-EAC-009-A_LACB09A \\
\hline CRB109 45-90 um; Calcite & Calcite & RELAB Spectral Library & CB-EAC-009-B_LACB09 \\
\hline CRB111 <45 um; Calcite & Calcite & RELAB Spectral Library & CB-EAC-011-A_LACB11A \\
\hline CRB112 <45 um; Calcite & Calcite & RELAB Spectral Library & CB-EAC-012-A_LACB12A \\
\hline CRB112 45-90 um; Calcite & Calcite & RELAB Spectral Library & CB-EAC-012-B_LACB12 \\
\hline CRB113 <45 um; Manganocalcite & Calcite & RELAB Spectral Library & CB-EAC-013-A_LACB13A \\
\hline CRB113 45-90 um; Manganocalcite & Calcite & RELAB Spectral Library & CB-EAC-013-B_LACB13 \\
\hline CRB128 <45 um; Calcite & Calcite & RELAB Spectral Library & CB-EAC-063-A_BKR1CB063A \\
\hline CRB130 <45 um; Calcite & Calcite & RELAB Spectral Library & CB-EAC-064-A_BKR1CB064A \\
\hline CRB131 <45 um; Calcite & Calcite & RELAB Spectral Library & CB-EAC-065-A_BKR1CB065A \\
\hline PIG004; CaCO3 Synthetic & Calcite & RELAB Spectral Library & EC-EAC-004_LAEC04 \\
\hline Calcite ; Calcite & Calcite & RELAB Spectral Library & GR-CMP-001_CAGR01 \\
\hline HYD-BLT-1a; Calcite & Calcite & RELAB Spectral Library & HM-JFM-001_C1HM01 \\
\hline Lane calcite $63-90$ & Calcite & RELAB Spectral Library & JB-JLB-550_BKR1JB550 \\
\hline Lane calcite $90-125$ & Calcite & RELAB Spectral Library & JB-JLB-551_1101S551 \\
\hline Lane calcite $125-180$ & Calcite & RELAB Spectral Library & JB-JLB-552_1101S552 \\
\hline Lane calcite $180-250$ & Calcite & RELAB Spectral Library & JB-JLB-553_1101S553 \\
\hline Lane calcite $250-355$ & Calcite & RELAB Spectral Library & JB-JLB-554_1101S554 \\
\hline Lane calcite $355-500$ & Calcite & RELAB Spectral Library & JB-JLB-555_1101S555 \\
\hline Mazada calcite 45-75 um & Calcite & RELAB Spectral Library & JB-JLB-E57-B_BKR1JBE57 \\
\hline Mazada calcite 75-90 um & Calcite & RELAB Spectral Library & JB-JLB-E57-C_BKR1JBE57C \\
\hline Mazada calcite 90-125 um & Calcite & RELAB Spectral Library & JB-JLB-E57-D_BKR1JBE57D \\
\hline Mazada calcite $125-250 \mathrm{um}$ & Calcite & RELAB Spectral Library & JB-JLB-E57-E_BKR1JBE57E \\
\hline Mazada calcite $<250$ um & Calcite & RELAB Spectral Library & JB-JLB-E57-F_BKR1JBE57F \\
\hline Big Timber calcite $<45$ um & Calcite & RELAB Spectral Library & JB-JLB-E58-A_BKR1JBE58A \\
\hline Big Timber calcite $45-75$ um & Calcite & RELAB Spectral Library & JB-JLB-E58-B_BKR1JBE58 \\
\hline Big Timber calcite 75-90 um & Calcite & RELAB Spectral Library & JB-JLB-E58-C_BKR1JBE58C \\
\hline
\end{tabular}


Table A.4. continued.

Big Timber calcite 90-125 um

Big Timber calcite 125-250 um

Big Timber calcite $<250$ um

Red Calcite

Calcite $\mathrm{CaCO} 3$

Calcite $\mathrm{CaCO} 3$

Calcite $\mathrm{CaCO} 3$

Calcite $\mathrm{CaCO} 3$

Calcite $\mathrm{CaCO} 3$

Calcite $\mathrm{CaCO} 3$

Calcite $\mathrm{CaCO} 3$

Calcite $\mathrm{CaCO} 3$

Calcite $\mathrm{CaCO} 3$

Calcite $\mathrm{CaCO} 3$

CRB103 <45 um; Dolomite

CRB103 45-90 um; Dolomite

CRB117 <45 um; Dolomite

Dolomite

Dolomite HS102

Fe dolomite $<125$ um

Selasvann dolomite $<45$ um

Selasvann dolomite 45-75 um

Selasvann dolomite 75-90 um

Selasvann dolomite 90-125 um

Selasvann dolomite 125-250 um

Selasvann dolomite $<250$ um

6521 Dolomite

6523 Dolomite

6528 Dolomite

6529 Dolomite

Dolomite $\mathrm{CaMg}(\mathrm{CO} 3) 2$

Dolomite $\mathrm{CaMg}(\mathrm{CO} 3) 2$

Dolomite $\mathrm{CaMg}(\mathrm{CO} 3) 2$

Dolomite $\mathrm{CaMg}(\mathrm{CO} 3) 2$

Dolomite $\mathrm{CaMg}(\mathrm{CO} 3) 2$

Dolomite $\mathrm{CaMg}(\mathrm{CO} 3) 2$

Dolomite $\mathrm{CaMg}(\mathrm{CO} 3) 2$

Dolomite $\mathrm{CaMg}(\mathrm{CO} 3) 2$

Dolomite $\mathrm{CaMg}(\mathrm{CO} 3) 2$

MGC 25-45 um; Magnesite

MGC 45-63 um; Magnesite

MGC 63-75 um; Magnesite

MGC 75-106 um; Magnesite

MGC 106-125 um; Magnesite

MGC 125-150 um; Magnesite

MGC 45-75 um; Magnesite

CRB106 <45 um; Magnesite

CRB106 45-90 um; Magnesite

CRB208 <45 um; Hydromagnesite

CRB144 <45 um; Magnesite

Brumado Bahia magnesite $<45$ um

Brumado Bahia magnesite 45-75 um

Brumado Bahia magnesite 75-125 um

Brumado Bahia magnesite $<125$ um
Calcite

Calcite

Calcite

Calcite

Calcite

Calcite

Calcite

Calcite

Calcite

Calcite

Calcite

Calcite

Calcite

Calcite

Dolomite

Dolomite

Dolomite

Dolomite

Dolomite

Dolomite

Dolomite

Dolomite

Dolomite

Dolomite

Dolomite

Dolomite

Dolomite

Dolomite

Dolomite

Dolomite

Dolomite

Dolomite

Dolomite

Dolomite

Dolomite

Dolomite

Dolomite

Dolomite

Dolomite

Magnesite

Magnesite

Magnesite

Magnesite

Magnesite

Magnesite

Magnesite

Magnesite

Magnesite

Magnesite

Magnesite

Magnesite

Magnesite

Magnesite

Magnesite
RELAB Spectral Library RELAB Spectral Library RELAB Spectral Library RELAB Spectral Library ECOSpeclib - JPL ECOSpeclib - JPL ECOSpeclib - JPL ECOSpeclib - JPL ECOSpeclib - JPL ECOSpeclib - JPL ECOSpeclib - JPL ECOSpeclib - JPL ECOSpeclib - JPL ECOSpeclib - JPL RELAB Spectral Library RELAB Spectral Library RELAB Spectral Library RELAB Spectral Library USGS Spectral Library Version RELAB Spectral Library RELAB Spectral Library RELAB Spectral Library RELAB Spectral Library RELAB Spectral Library RELAB Spectral Library RELAB Spectral Library RELAB Spectral Library RELAB Spectral Library RELAB Spectral Library RELAB Spectral Library ECOSpeclib - JPL ECOSpeclib - JPL ECOSpeclib - JPL ECOSpeclib - JPL ECOSpeclib - JPL ECOSpeclib - JPL ECOSpeclib - JPL ECOSpeclib - JPL ECOSpeclib - JPL RELAB Spectral Library RELAB Spectral Library RELAB Spectral Library RELAB Spectral Library RELAB Spectral Library RELAB Spectral Library RELAB Spectral Library RELAB Spectral Library RELAB Spectral Library RELAB Spectral Library RELAB Spectral Library RELAB Spectral Library RELAB Spectral Library RELAB Spectral Library RELAB Spectral Library
JB-JLB-E58-D_BKR1JBE58D

JB-JLB-E58-E_BKR1JBE58E

JB-JLB-E58-F_BKR1JBE58F PC-CMP-055_C1PC55

coarse.tir.c-3e.jpl.nicolet.spectrum coarse.tir.calcite_1.jhu.nicolet.spectrum coarse.tir.calcite_2.jhu.nicolet.spectrum fine.tir.c-3d.jpl.nicolet.spectrum fine.tir.c-3e.jpl.nicolet.spectrum fine.tir.calcite_1.jhu.nicolet.spectrum fine.vswir.c-3a.jpl.beckman.spectrum medium.tir.c-3d.jpl.nicolet.spectrum medium.tir.c-3e.jpl.nicolet.spectrum medium.vswir.c-3a.jpl.beckman.spectrum CB-EAC-003-A_LACB03A CB-EAC-003-B_LACB03

CB-EAC-017-A_BKR1CB017A

CC-JFM-005-B_F1CC05

Dolomite_HS102.3B_NIC4bbb_RREF

JB-JLB-779_BKR1JB779

JB-JLB-E61-A_BKR1JBE61A

JB-JLB-E61-B_BKR1JBE61

JB-JLB-E61-C_BKR1JBE61C

JB-JLB-E61-D_BKR1JBE61D

JB-JLB-E61-E_BKR1JBE61E

JB-JLB-E61-F_BKR1JBE61F

SH-SJG-077_NASH77

SH-SJG-078_NASH78

SH-SJG-079_NASH79

SH-SJG-080_NASH80

coarse.tir.c-5a.jpl.nicolet.spectrum coarse.tir.dolomite_1.jhu.nicolet.spectrum coarse.tir.dolomite_2.jhu.nicolet.spectrum coarse.tir.dolomite_3.jhu.nicolet.spectrum fine.tir.c-5a.jpl.nicolet.spectrum fine.tir.c-5c.jpl.nicolet.spectrum medium.tir.c-5a.jpl.nicolet.spectrum medium.tir.c-5c.jpl.nicolet.spectrum solid.tir.dolomite_3.jhu.nicolet.spectrum BE-JFM-223_BKR1BE223

BE-JFM-224_BKR1BE224

BE-JFM-225_BKR1BE225

BE-JFM-226_BKR1BE226

BE-JFM-227_BKR1BE227

BE-JFM-228_BKR1BE228

BE-JFM-231_BKR1BE231

CB-EAC-006-A_LACB06A

CB-EAC-006-B_LACB06

CB-EAC-028-A_LACB28A

CB-EAC-070-A_BKR1CB070A

JB-JLB-946-A_BKR1JB946A

JB-JLB-946-B_BKR1JB946

JB-JLB-946-C_BKR1JB946C

JB-JLB-946-E_BKR1JB946E 


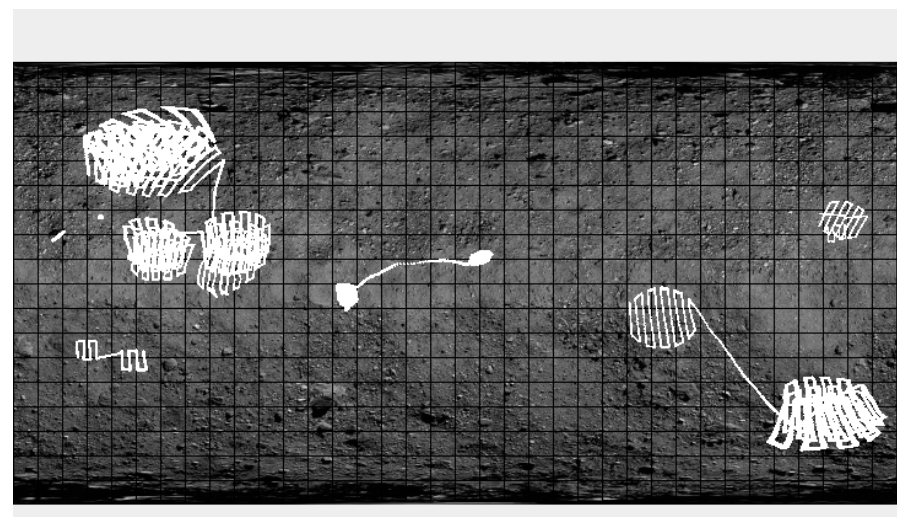

Fig. A.1. OVIRS footprints from the Recon A phase of the OSIRISREx mission overlaid on a Bennu base map, which extends from $-90^{\circ}$ to $90^{\circ} \mathrm{N}$ and $0^{\circ}$ to $360^{\circ} \mathrm{E}$. There are 15858 footprints shown on this map, which were analyzed for organic spectral features in this study.
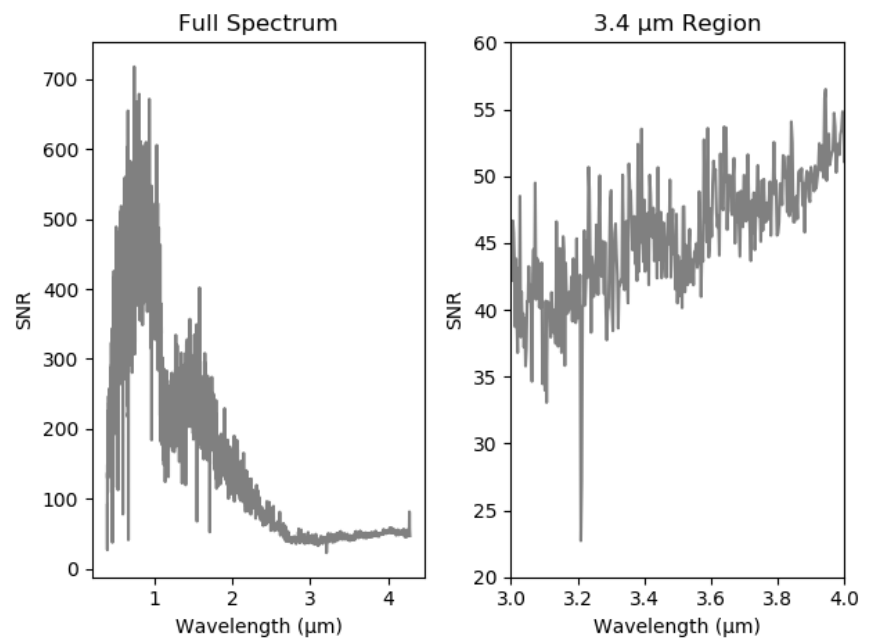

Fig. A.2. OVIRS S/N near $3.4 \mu \mathrm{m}$, average for Recon A.
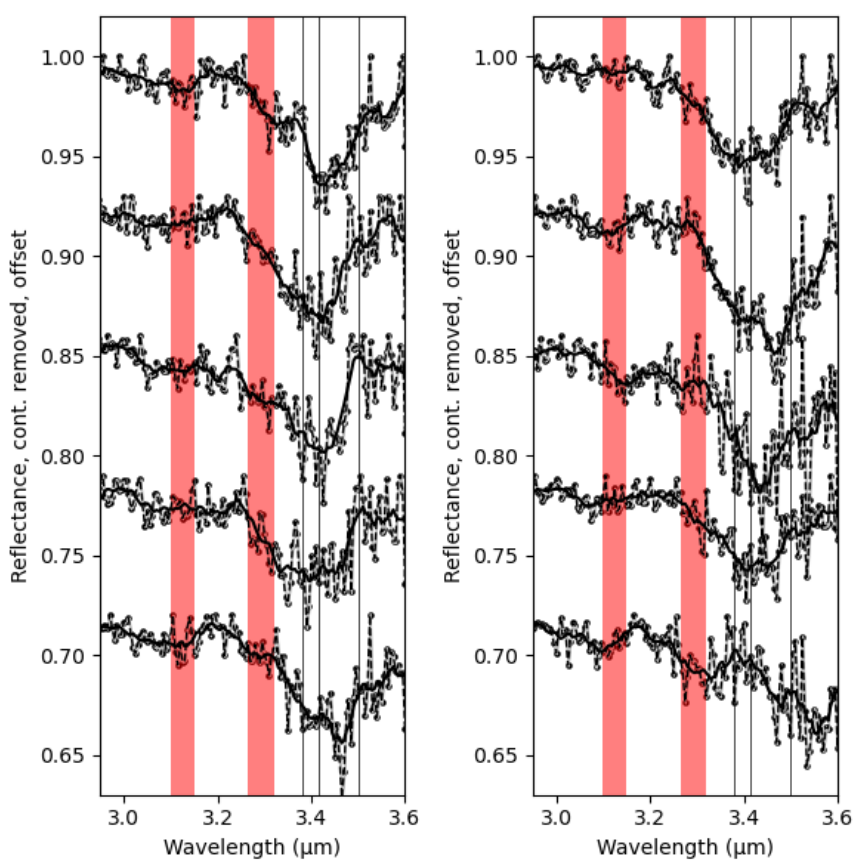

Fig. A.3. Example Bennu spectra that have a "shoulder" near $3.3 \mu \mathrm{m}$ that may be indicative of aromatic $\mathrm{CH}$. Vertical lines at 3.10, 3.275, $3.38,3.42$, and $3.50 \mu \mathrm{m}$ indicate positions of possible absorption features.
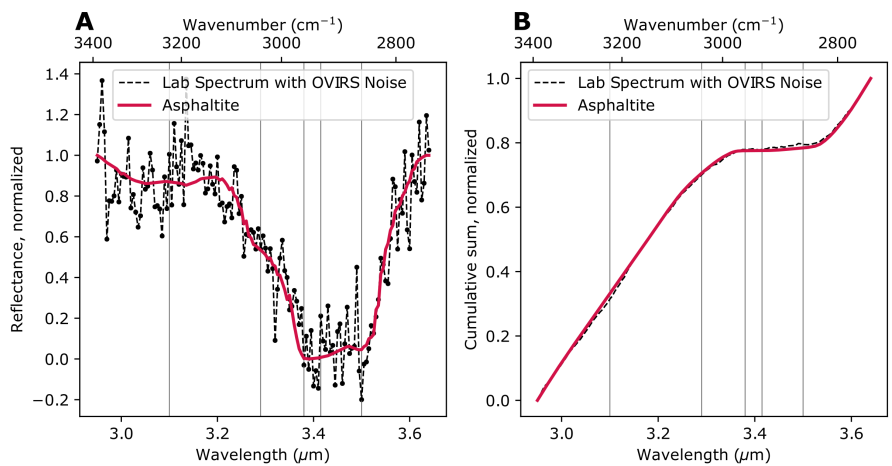

Fig. A.4. Example of the two fitting methods developed in two previous studies (Kaplan et al. 2020; Ferrone et al. 2021): (a) linear leastsquares fitting of normalized data and (b) a Kolmogorov-Smirnov fitting method, in which we search for the maximum distance between the two curves. The black curves are laboratory data with noise added to simulate OVIRS noise, and the red curves are laboratory data at their original $\mathrm{S} / \mathrm{N}$. Vertical lines at $3.10,3.275,3.38,3.42$, and $3.50 \mu \mathrm{m}$ indicate positions of possible absorption features. 